\title{
Planetary Gearbox Fault Diagnosis Using Envelope Manifold Demodulation
}

\author{
Weigang Wen, ${ }^{1,2}$ Robert X. Gao, ${ }^{2,3}$ and Weidong Cheng ${ }^{1}$ \\ ${ }^{1}$ School of Mechanical, Electronic and Control Engineering, Beijing Jiaotong University, Beijing 100044, China \\ ${ }^{2}$ Department of Mechanical Engineering, University of Connecticut, Storrs, CT 06269, USA \\ ${ }^{3}$ Mechanical and Aerospace Engineering Department, Case Western Reserve University, Cleveland, OH 44106, USA \\ Correspondence should be addressed to Weigang Wen; wgwen@bjtu.edu.cn
}

Received 4 July 2015; Revised 16 September 2015; Accepted 27 September 2015

Academic Editor: José V. Araújo dos Santos

Copyright (C) 2016 Weigang Wen et al. This is an open access article distributed under the Creative Commons Attribution License, which permits unrestricted use, distribution, and reproduction in any medium, provided the original work is properly cited.

The important issue in planetary gear fault diagnosis is to extract the dependable fault characteristics from the noisy vibration signal of planetary gearbox. To address this critical problem, an envelope manifold demodulation method is proposed for planetary gear fault detection in the paper. This method combines complex wavelet, manifold learning, and frequency spectrogram to implement planetary gear fault characteristic extraction. The vibration signal of planetary gear is demodulated by wavelet enveloping. The envelope energy is adopted as an indicator to select meshing frequency band. Manifold learning is utilized to reduce the effect of noise within meshing frequency band. The fault characteristic frequency of the planetary gear is shown by spectrogram. The planetary gearbox model and test rig are established and experiments with planet gear faults are conducted for verification. All results of experiment analysis demonstrate its effectiveness and reliability.

\section{Introduction}

Planetary gear systems are widely used in industrial machinery because they have advantages of large transmission ratios, strong load-bearing capacity, and high transmission efficiency [1]. In practical industrial fields, the planetary gear system whose structure is more complicated than fixed-shaft gearbox is subject to be damaged under heavy load and tough working conditions. Accurate fault detection of planetary gearbox is important to reduce unscheduled machine downtime and avoid catastrophic accidents [2]. Vibration signal analysis which was used extensively in various machine condition monitoring techniques has become one of the most important methods applied for planetary gearbox fault diagnostics.

The planetary gear system is composed of sun gear, planet gears, ring gear, planet carrier, shaft, and bearings. Usually, the ring gear is stationary, the sun gear rotates around its own center, and the planet carrier rotates under very low speeds because of the large transmission ratio. Multiple planet gears, which mesh simultaneously with the sun gear and ring gear, not only rotate around their own center but also revolve around the center of the sun gear [3]. Such behaviors cause the vibration signals of the planetary gearbox with different meshing phases and varied propagation paths [4]. The unique behavior mode of planetary gearbox presents a challenge to fault diagnosis of planetary gear system. Studies on the fault diagnosis of planetary gearboxes are gaining more attention of the researchers [5].

The fault diagnosis of planetary gearboxes comes from various methodologies. Modeling methods are useful in condition monitoring. Tooth pitting and crack on the sun gear of planetary gearboxes were modeled and the influence of faults on the gear mesh stiffness was analyzed in [6]. An approach based on physical models to detect damage of a planetary gearbox in a helicopter transmission system was proposed in [7]. The spectral structure of vibration signal of planetary gearbox in different health conditions was investigated and vibration signal models of gear damage were proposed in [8]. But the most popular fault diagnosis of 
planetary gearbox is signal processing methods, for example, time-domain methods, frequency-domain methods, timefrequency-domain methods, and other signal processing methods. Time-domain signal processing methods, such as statistical indicators and time synchronous averaging, are relatively easy and direct compared to other methods. A technique for estimating the time-domain average of the tooth meshing vibration of individual planet gears and of the sun gear was demonstrated in [9]. Two special diagnostic parameters in time-domain for detecting faults of planetary gearbox were studied in [10]. In frequency-domain, the Fourier series of the vibration data from a planetary gearbox was explored and the source of the asymmetry phenomena in the frequency spectra was analyzed in [1]. The additional sidebands in frequency spectra produced by planet carrier torque modulations masking potentially the sidebands of fault in planetary gearboxes were investigated and a frequency-domain method to eliminate the effects of structural-path-caused amplitude changes was presented in [11]. The time-frequency-domain methods, such as WignerVille distribution and wavelets, are developed to diagnose planetary gearboxes. The Wigner-Ville distribution of the simulated signals of fault modes in planetary gearboxes was studied in [12]. A method based on adaptive Morlet wavelets and singular value decomposition was applied to extract impulse features of a planetary gearbox in [13]. Reference [14] illustrated the spectral kurtosis for detecting a tooth crack on the ring in the planetary gearbox of a wind turbine. The cyclostationary properties of planetary gearbox vibration signals were investigated and a fault diagnosis based on spectral coherence map was developed in [15]. These literatures demonstrated the different effects of signal processing methods. The time-domain and frequencydomain methods just depict one aspect of the signal. The characteristic in time-domain and frequency-domain cannot be drawn simultaneously. Time-frequency-domain methods are generally much more effective than the other two. But it is better to combine them with other methods to implement demodulating and denoising.

The vibration signals of mechanical systems, especially for ones with fault, often show mutation, nonlinearity, and nonstationarity [16]. With the development of nonlinear dynamic theories, a number of nonlinear parameters and methods have been applied to machine condition monitoring and fault diagnosis [17-19]. Manifold learning especially is adopted to implement nonlinear dimensionality reduction for revealing the nonlinear intrinsic characteristics of vibration signal. The signal processing technique and manifold learning method are combined to strengthen the fault features of the rotating machine and reduce the impact of noise [20]. The manifold learning algorithm on the time-frequency distributions or the time-scale distributions generated manifold signature of fault to enhance nonlinear fault-induced components and suppress in-band noise in [21-23].

In planetary gearbox, the vibration signals from multiple planet gears may couple with each other leading the faulty components to be neutralized and cancelled [24]. Especially in practical industrial applications, the vibration signal is contaminated by background noise. Here, in order to extract the fault feature of planetary gear from the noisy vibration signal, the methodology implementing decoupling, demodulating, and denoising is investigated. Because of the demodulation capability of wavelet envelope and the denoising ability of manifold learning $[20,25]$, these methods are integrated to achieve demodulating and denoising of the planetary gear vibration signal simultaneously in this paper.

The rest of the paper is organized as follows. In the second section, the wavelet envelope manifold demodulation method is introduced for fault diagnosis of planetary gearbox. Then in the third section, the vibration models and simulation experiments of the planetary gearbox in faulty condition are studied. In the fourth section, the practical vibration signals from planetary gearbox rig are analyzed to testify the effectiveness of the proposed method. The conclusions are finally presented in the fifth section.

\section{Principle and Methodology}

2.1. Framework of Methodology. In fault diagnosis of planetary gears, the accelerometers are fixed on the gearbox casing to collect vibration signals. The relative locations of sun-planet and planet-ring meshing with respect to the accelerometers are time-varying, which result in amplitude modulation (AM) effect on the meshing vibration signal. Time variation of meshing stiffness or the errors of the planetary gear also causes frequency modulation (FM) of the meshing vibration signal. The gear damage generates both $\mathrm{AM}$ and FM of the meshing vibration signal. These effects increase the complexity of the signal. Furthermore, there exists the random background noise in every frequency band for practical fault diagnosis of planetary gear in industrial fields. The noise can blight the signal at meshing frequency. So, extracting the meshing signal, reducing the noise effect in meshing frequency band, and demodulating the meshing vibration signal are the critical issues for fault diagnosis of planetary gearbox.

The enveloping analysis of resonance is a powerful tool to achieve demodulation of the signal of rotating machine. In the traditional way, a filter with resonance frequency and specific pass band is utilized to preprocess the vibration signal, and then the envelope of the resonance is drawn out by Hilbert transform. Here, a synthetic methodology that combines the complex wavelet transform, manifold learning method, and spectral analysis is proposed to demodulate and denoise the meshing vibration signal of planetary gears. The methodology is shown in Figure 1. At first, the continuous wavelet transform (CWT) with complex Morlet wavelet base is utilized to demodulate the vibration signal over scales to obtain the wavelet envelopes. Then, the meshing vibration sensitive indicator is built to select the specific scale band which corresponds to meshing frequency band. The first two steps both extract the envelope and filter out the noise not within the meshing frequency band. Thirdly, the manifold learning algorithm is introduced to extract the envelope manifold from the wavelet envelopes at the selected scales to reduce the in-band noise effect and reveal the envelope structure of the meshing vibration signal. Finally, the fault 

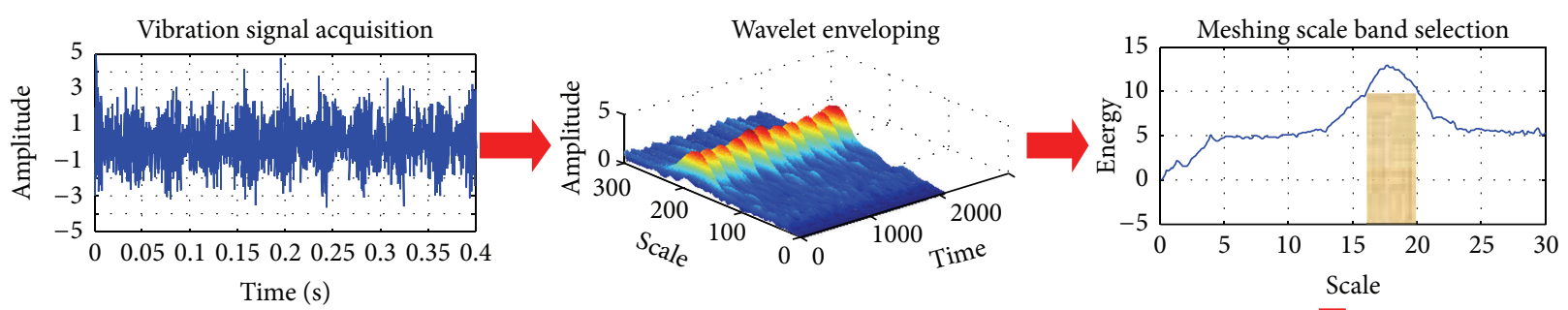

Time (s)
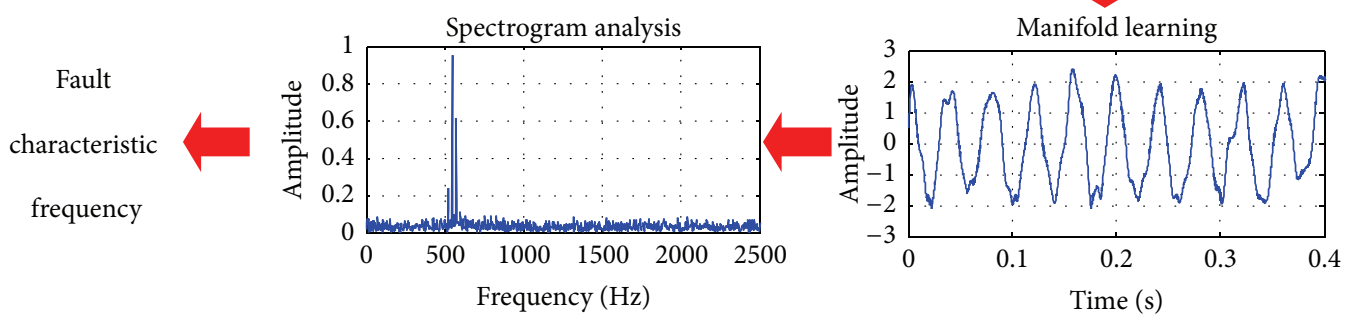

FIGURE 1: The process diagram of the methodology.

characteristic frequency is discovered by frequency spectrogram analysis.

2.2. Complex Wavelet Enveloping. It is well known that the wavelet transform can decompose a signal onto a time-scale plane [25]. The wavelet transform of a signal is equal to a set of band-pass filters with certain frequencies relevant to the scales. Continuous wavelet transform (CWT) is defined as the convolution of signal $x(t)$ with the complex conjugate of a set of wavelets. Here, complex wavelet $\varphi(t)$ is applied. The complex wavelet has the analytic property. It is defined as

$$
\varphi(t)=\varphi_{R}(t)+j \varphi_{I}(t)=\varphi_{R}(t)+j \mathscr{H}\left[\varphi_{R}(t)\right],
$$

where $\varphi_{R}(t)$ and $\varphi_{I}(t)$ represent the real and imaginary parts of the complex wavelet and $\mathscr{H}[]$ is the Hilbert transform. Usually, the complex Morlet wavelet is used as the base wavelet for the vibration signal of rotating machine. The complex Morlet wavelet is defined as

$$
\varphi(t)=\frac{1}{\sqrt{\pi f_{b}}} e^{-t^{2} / f_{b}} e^{j 2 \pi f_{c} t},
$$

where $f_{b}$ is the bandwidth parameter and $f_{c}$ is the wavelet center frequency. Here, the parameter optimization of the Morlet wavelet is not the research focus of this paper. The parameters are set as $f_{b}=1$ and $f_{c}=2$ by which the satisfied results can be achieved. The base wavelet with specific scale $s$ is used to extract the component of frequency $f$ in the signal. The relationship can be written as

$$
f=\frac{f_{c}}{s \Delta},
$$

where $\Delta$ is the sampling period. From formula (3), the meshing frequency of the planetary gears can be estimated according to the special indicator at the scale. The wavelet transform of the signal based on complex wavelet can be written as

$$
\begin{aligned}
W_{C}(s, \tau) & =W_{R}(s, \tau)+j W_{I}(s, \tau) \\
& =W_{R}(s, \tau)+j \mathscr{H}\left[W_{R}(s, \tau)\right] .
\end{aligned}
$$

According to the property of Hilbert transform, the envelope of signal at scale $s$ can be computed from the modulus of the wavelet coefficients as

$$
E_{W}(s, \tau)=\left\|W_{C}(s, \tau)\right\|=\sqrt{W_{R}^{2}(s, \tau)+W_{I}^{2}(s, \tau)} .
$$

Through complex wavelet transformation and scale band selection, the band-pass filtering and enveloping can be achieved simultaneously.

2.3. Meshing Scale Band Selection. In the enveloping technique for fault diagnosis of rotating machine, the critical issue is to estimate the resonance frequency of the structure. In current literatures different indicators have been applied for decision of resonance modes. The spectral kurtosis (SK) is an effective measure to estimate the resonance frequency of impulsive components in vibration signal [26-29]. The smoothness index (SI) defined as the ratio of the arithmetic mean to the geometric mean is also utilized to measure the impulsiveness of the power spectrum of wavelet coefficient at each scale in [30]. After complex wavelet filtering and enveloping, the energy of vibration signal envelope concentrated within meshing frequency band more than the other band. The mean envelope energy can be adopted as the indicator for selection of meshing scale band. For the wavelet envelope of the signal $x(t)$, the mean energy (ME) of wavelet envelope at scale $s$ is defined as

$$
\operatorname{ME}(s)=\frac{\sum_{\tau} E_{W}(s, \tau) E_{W}^{*}(s, \tau)}{N},
$$

where $E_{W}^{*}(s, \tau)$ is the complex conjugate of $E_{W}(s, \tau)$ and $N$ is number of sample points. When the ME, SK, and SI in 
frequency-domain are considered as indicators for selecting the appropriate scale for the meshing frequency in the data simulations and practical experiments, it is revealed that the $\mathrm{ME}$ is most suitable to select scale corresponding to the meshing frequency.

The scale corresponding to the global maximum of the indicator is defined as central scale $s_{C}$, which is utilized to evaluate the planetary gear meshing central frequency. The wavelet envelopes in meshing scale band, which construct a high-dimensional data space, contain the fault information in the meshing frequency band. The meshing scale band can be divided by the left limit $s_{L}$ and right limit $s_{R}$ around the central scale $s_{C}$. The left limit $s_{L}$ and right limit $s_{R}$ can be determined by the indicator value reducing to $1 / \sqrt{2}$ of the maximum value of the indicator at the central scale $s_{C}$. So the scale band for the meshing frequency band can be defined as $s_{B}=\left[s_{L}, \ldots, s_{C}, \ldots, s_{R}\right]$.

The fault-induced impacts modulating the meshing frequency form the manifold structure embedded in the wavelet envelopes at this scale band. There is also another background noise within meshing frequency band. The in-band noise will corrupt the embedded manifold structure. Here, manifold learning is applied to retain the envelope manifold of the fault impacts while leaving out the in-band noise. And the $n$-dimension $\left(n=R-L+1\right.$, where $R$ is the index of $s_{R}$ and $L$ is the index of $s_{L}$ ) data space can be reduced to $m$-dimension $(m \ll n)$ by mean of the nonlinear dimensionality reduction for posttreatment.

2.4. Manifold Learning. There are several algorithms of manifold learning. The techniques of Isomap and locally linear embedding (LLE) were firstly proposed. Isomap is a global method because its embedding is based upon the geodesic distances between all pairs of points, while LLE is a local method because its embedding is based upon the relationship of each data point to its neighboring points $[31,32]$. Laplacian eigenmap, Hessian eigenmap, local tangent alignment, diffusion map, and several other techniques were introduced.

A local approach is computationally more efficient than a global approach because the former involves sparse matrices and is more generally applicable to different types of manifolds. Laplacian eigenmap, which is a typical local approach, is applied to extract the manifold structure from the wavelet envelopes at planetary gear meshing frequency band because of its efficiency and robustness to the noise [33].

The methodology of Laplacian eigenmap algorithm consists of three steps: (1) construct $K$ nearest neighbor graph on the set of sample points; (2) approximate the local manifold geometry within the neighborhood of each sample point; (3) minimize a global error function to obtain the global embedding [34].

Here, the number of nearest neighbor is set as $K=$ 40 , the reduced dimension is set as $m=2$, and the first dimension data are taken as the signal for the next step of frequency spectrogram analysis for fault characteristic frequency extraction because of its minimum reconstruction error.

\section{Simulated Signal Analysis}

3.1. Simulation of Faulty Planet Gear. The planet gears mesh with ring gear and sun gear simultaneously. When there are faults on a planet gear, the meshing location of the faulty tooth and mating gear changes with the planet carrier rotation. Thus this changes the transfer path of the vibration signal of faulty tooth to the sensor and generates an extra AM effect on the meshing vibration signal. The meshing vibration amplitudes sampled by the sensor are modulated by the faulty tooth and the varied propagations synchronously [8]. Here, the faulty planet gear is analyzed. The faulty planet gear vibration signal can be written as

$$
\begin{aligned}
x(t)= & {\left[1-C \cos \left(2 \pi f_{\text {carrier }} t\right)\right] } \\
& \cdot\left[1+A \cos \left(2 \pi f_{\text {planet }} t+\emptyset\right)\right] \\
& \cdot \cos \left[2 \pi f_{\text {mesh }} t+B \sin \left(2 \pi f_{\text {planet }} t+\varphi\right)+\theta\right],
\end{aligned}
$$

where $x(t)$ is vibration signal in time-domain, $f_{\text {carrier }}$ is planet carrier rotation frequency which represents propagation modulation, $f_{\text {planet }}$ is planet gear rotation frequency which represents faulty planet gear modulation, $f_{\text {mesh }}$ is meshing frequency, and $\emptyset, \varphi$, and $\theta$ are the initial phases of AM, FM, and meshing, respectively. $A, B$, and $C$ are the modulation amplitudes. After amplitude demodulation, the envelope of the meshing vibration signal is

$$
\begin{aligned}
y(t)=1 & -C \cos \left(2 \pi f_{\text {carrier }} t\right)+A \cos \left(2 \pi f_{\text {planet }} t+\emptyset\right) \\
& +\frac{1}{2} A C \cos \left[2 \pi\left(f_{\text {planet }}+f_{\text {carrier }}\right) t+\emptyset\right] \\
& +\frac{1}{2} A C \cos \left[2 \pi\left(f_{\text {planet }}-f_{\text {carrier }}\right) t+\emptyset\right] .
\end{aligned}
$$

So the envelope amplitude spectrum is

$$
\begin{aligned}
Y(f)= & \delta(f)+C \delta\left(f-f_{\text {carrier }}\right)+A \delta\left(f-f_{\text {planet }}\right) \\
& +\frac{1}{2} A C \delta\left[f-\left(f_{\text {planet }}+f_{\text {carrier }}\right)\right] \\
& +\frac{1}{2} A C \delta\left[f-\left(f_{\text {planet }}-f_{\text {carrier }}\right)\right]
\end{aligned}
$$

The simulation parameters of the planetary gearbox are shown in Table 1. For comparison, the time-domain diagram and frequency spectrogram are adopted to analyze the vibration signals at first. Then the envelope manifold demodulation is utilized to illustrate the advantage of this method.

The simulated vibration signal in time-domain is shown in Figure 2(a). The frequency spectrogram of the signal is shown in Figure 2(b). The meshing frequency of $437.5 \mathrm{~Hz}$ and the sideband can be seen in the spectrogram. It is not very clear to identify the planet carrier rotation frequency and the planet gear rotation frequency.

In envelope manifold demodulation, a series of scales of wavelet ranging from 1 to 30 are chosen to cover the meshing frequency of all the planetary gears. An increment of 0.1 


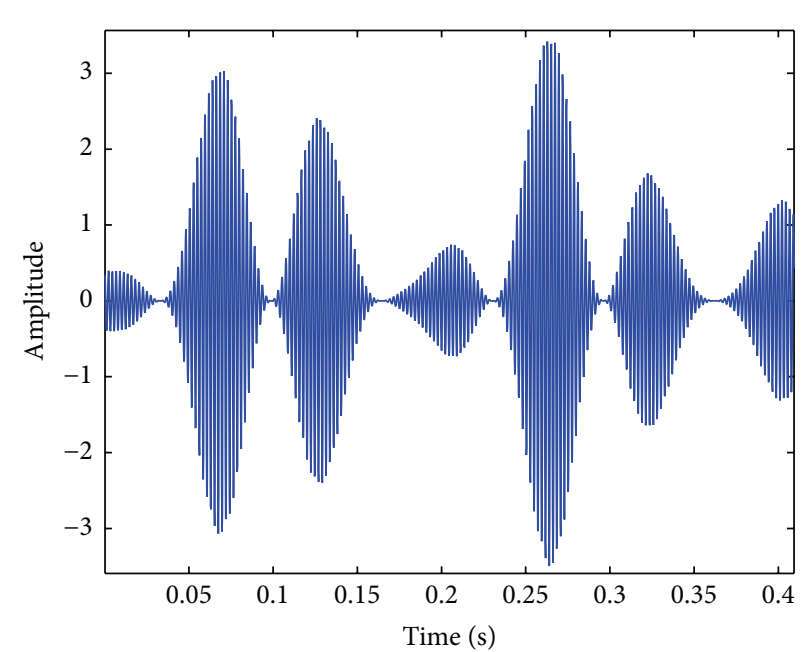

(a) The time-domain diagram

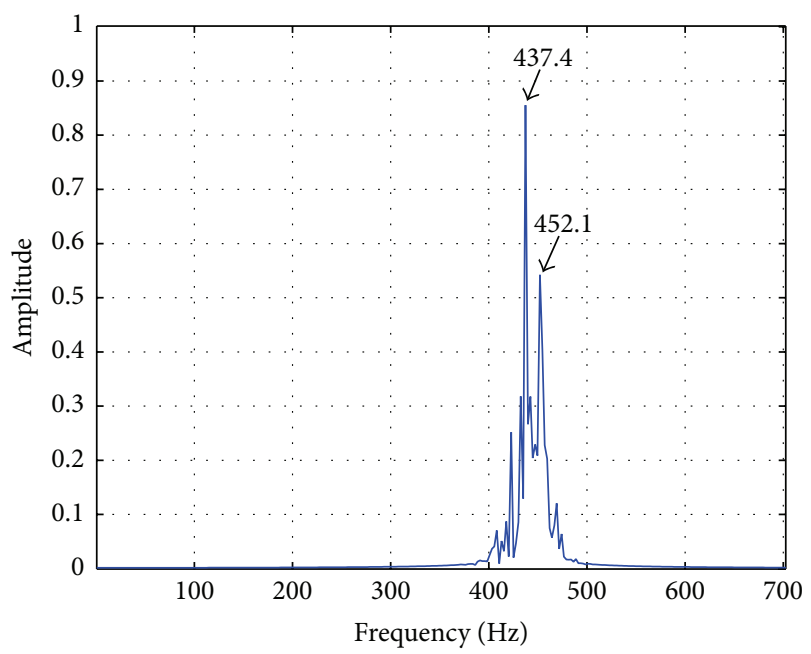

(b) The frequency spectrogram

FIGURE 2: The time-domain diagram and frequency spectrogram of simulated signal.

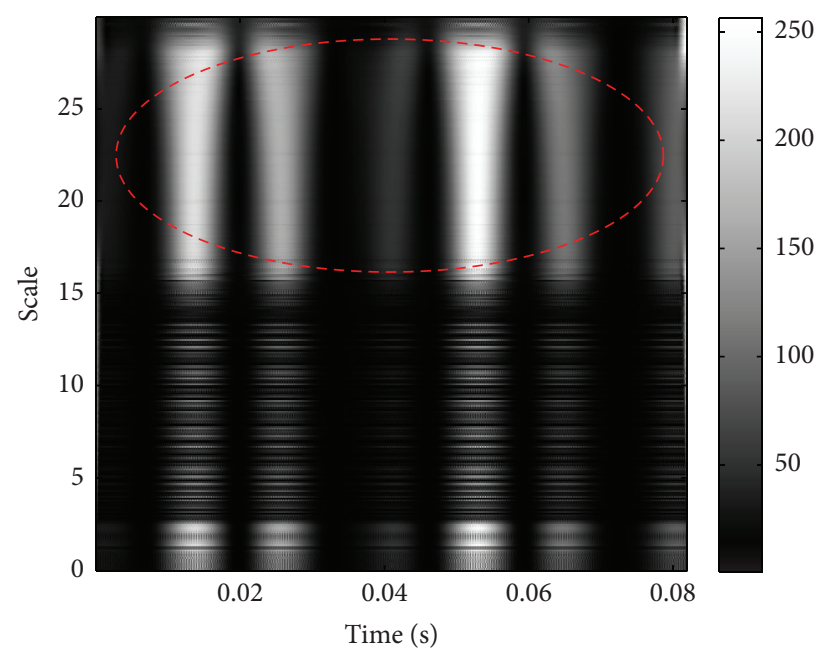

FIgURE 3: The wavelet envelope of simulated signal.

TABLE 1: The simulation parameters of faulty planet gear.

\begin{tabular}{lc}
\hline Parameters & Values \\
\hline AM magnitude $A$ & 1 \\
AM magnitude $C$ & 0.8 \\
FM magnitude $B$ & 0.5 \\
Meshing frequency $(\mathrm{Hz})$ & 437.5 \\
Planet carrier rotation frequency $(\mathrm{Hz})$ & 5.468 \\
Planet gear rotation frequency $(\mathrm{Hz})$ & 15.191 \\
\hline
\end{tabular}

for scale is employed in the data experiments. The wavelet envelopes are shown in Figure 3. The lighter color in the graph illustrates greater wavelet envelopes. So the area circled by the ellipse corresponds to the greatest wavelet envelopes. The meshing frequency computed by formula (3) is the central frequency of the circled area.
The mean energy (ME), smoothness index (SI), and spectral kurtosis (SK) of wavelet envelope are utilized to choose the proper indictor of meshing frequency for comparison. The ME, SI, and SK as indicators are shown in Figures 4(a), $4(\mathrm{~b})$, and 4 (c). The central scale, respectively, is $22.8,22.2$, and 29.0 according to the ME, SI, and SK. The corresponding frequency, respectively, is $438.5 \mathrm{~Hz}, 450.5 \mathrm{~Hz}$, and $344.8 \mathrm{~Hz}$. The result from $\mathrm{ME}$ is closest to the meshing frequency band which is circled by the ellipse in Figure 3. The indicator ME is most accurate for selection of meshing frequency band.

The wavelet envelope at the central scale is shown in Figure 5(a) and the power spectrum of the wavelet envelope is shown in Figure 5(b). The characteristic frequencies of the power spectrum are $4.89 \mathrm{~Hz}, 9.78 \mathrm{~Hz}, 14.66 \mathrm{~Hz}$, and $19.55 \mathrm{~Hz}$ corresponding to $f_{\text {carrier }}, f_{\text {planet }}-f_{\text {carrier }}, f_{\text {planet }}$, and $f_{\text {planet }}+f_{\text {carrier }}$. The characteristic frequency of faulty planet gear can be seen clearly on the spectrogram of the wavelet envelope because there is no in-band noise interference. 


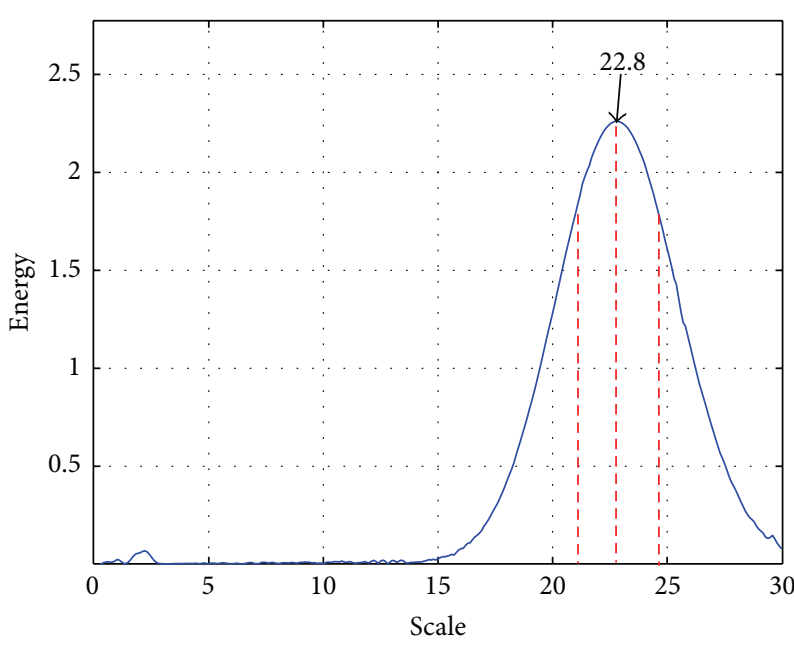

(a) The ME versus scale curve

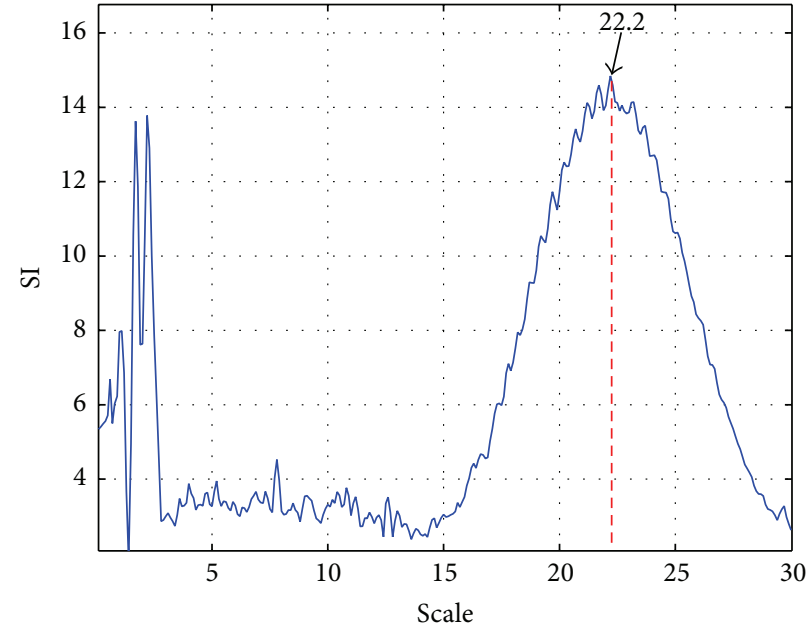

(b) The SI versus scale curve

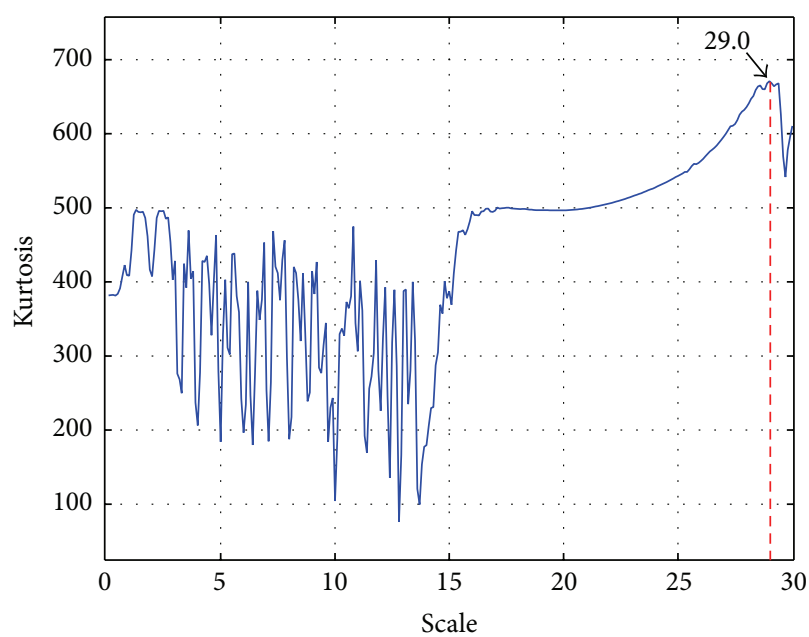

(c) The SK versus scale curve

FIGURE 4: The indicators versus scale curve of simulated signal.

The wavelet envelope is proved to be adept at demodulation of planetary gear vibration signal.

3.2. Simulation of Contaminated Signal. In order to simulate the contamination of the in-band noise, the random background noise is added to the simulated signal of the faulty planet gear model to generate the contaminated signal up to $\mathrm{SNR}=-10 \mathrm{~dB}$. Meanwhile, the meshing frequency is switched from $437.5 \mathrm{~Hz}$ to $492.0 \mathrm{~Hz}$ to testify the accuracy of selection of meshing frequency by ME.

For comparison, the amplitude demodulation methods based on empirical mode decomposition (EMD) and Hilbert transform are used to reveal the characteristic frequency of the planetary gears at first. It is well known that these methods are capable of demodulating the signal when it is not contaminated seriously by noise $[35,36]$. But when random background noise is added to the signal, the demodulation ability of these methods diminishes dramatically. When the signal is contaminated by noise with $S N R=-6 \mathrm{~dB}$, the EMD analysis results are shown in Figures 6(a) and 6(b). The fault characteristic frequency is not very clear in the spectrogram.

When the signal is contaminated by noise with SNR $=-8 \mathrm{~dB}$, the envelope and the frequency spectrogram of Hilbert transform after filtering are shown in Figures 7 (a) and 7(b). The fault characteristic frequency cannot be seen in the envelope spectrum.

In these methods, the EMD can be seen as a process of band filter, and the band-pass filter is applied prior to the Hilbert transform. The noise out of meshing frequency band is filtered out. But the in-band noise distorts the original vibration signal causing the invisibility of faulty characteristic frequency.

When the signal is contaminated by noise with SNR = $-10 \mathrm{~dB}$, the wavelet envelopes can be shown in Figure 8(a). There is so much noise pollution that the original signal cannot be recognized. The $\mathrm{ME}$ as indicator is shown in Figure 8 (b). The central scale 20.3 according to the ME is the central frequency of $492.6 \mathrm{~Hz}$. The results show that the indicator of $\mathrm{ME}$ is accurate and robust to the noise 


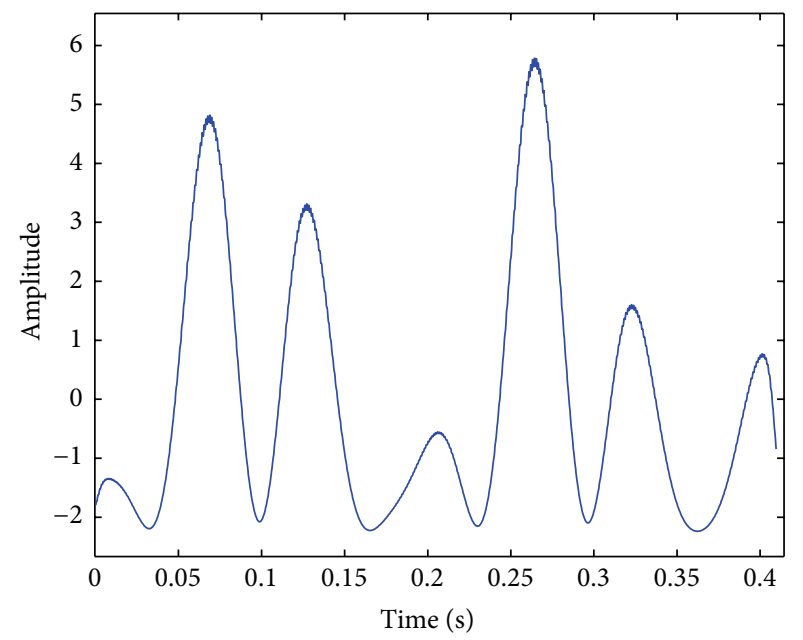

(a) The wavelet envelope

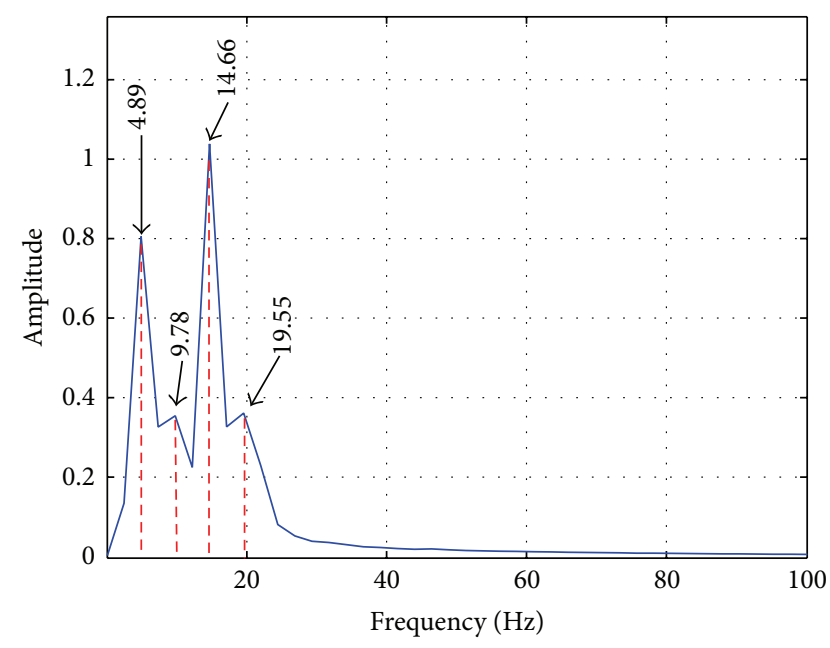

(b) The envelope frequency spectrogram

FIgURE 5: The wavelet envelope and spectrogram at the central scale of simulated signal.

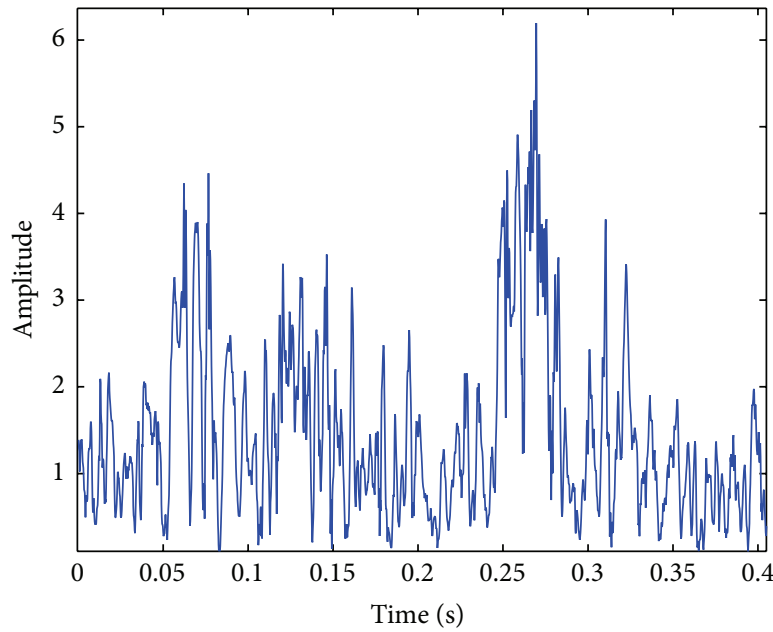

(a) An intrinsic mode function (IMF) of EMD decomposition

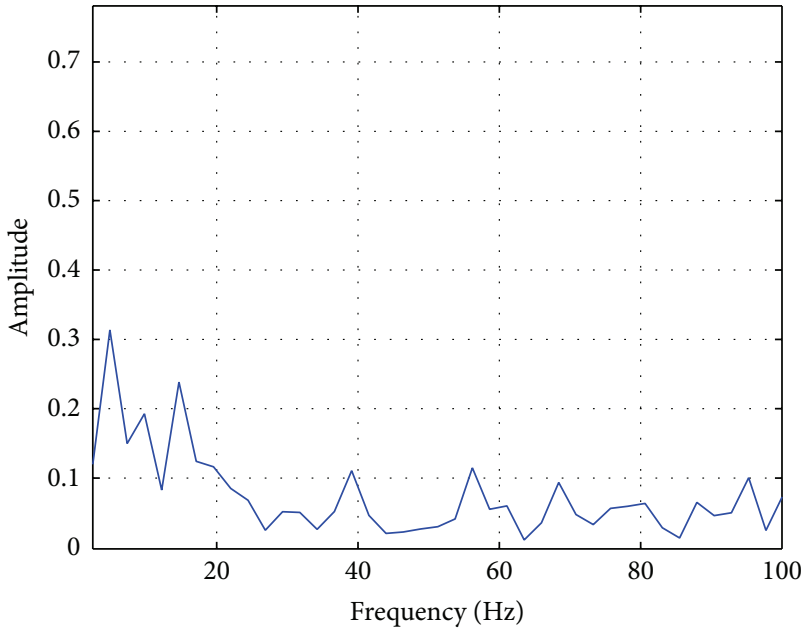

(b) The frequency spectrogram of the IMF

FIgURE 6: The EMD analysis result of contaminated signal with $\mathrm{SNR}=-6 \mathrm{~dB}$.

up to $\mathrm{SNR}=-10 \mathrm{~dB}$. After manifold learning, the wavelet envelope and the power spectrum are shown in Figures 8(c) and $8(\mathrm{~d})$. All the characteristic frequencies can be seen in the envelope manifold spectrogram. These data experiments fully demonstrate the superiority of the envelope manifold demodulation method.

\section{Experimental Signal Analysis}

4.1. Experiment Design. In this section, the cases of practical experiments are studied to verify the effectiveness of the envelope manifold demodulation methodology. The planetary gearbox test rig is shown in Figure 9. The planetary gear set in the gearbox is shown in Figure 10. The motor drives the input shaft of planetary gearbox through coupler. The input shaft is coupled to the sun gear, the ring gear is standstill, and the planet carrier is coupled to the output shaft which
TABLE 2: The planetary gearbox parameters.

\begin{tabular}{lc}
\hline Parameters & Values \\
\hline Planet gear teeth & 36 \\
Sun gear teeth & 28 \\
Ring gear teeth & 100 \\
Number of planet gears & 3 \\
\hline
\end{tabular}

drives the other load gearboxes and load motor. The rotation speed of the planetary gearbox input shaft is measured by the tachometer. The vibration signals are collected by the accelerometer mounted on the planetary gearbox. The experimental planet gears are shown in Figure 11. The parameters of planetary gearbox are listed in Table 2 .

Three experiments are designed for comparison. The first case is the experiment of the normal planet gear, the second 


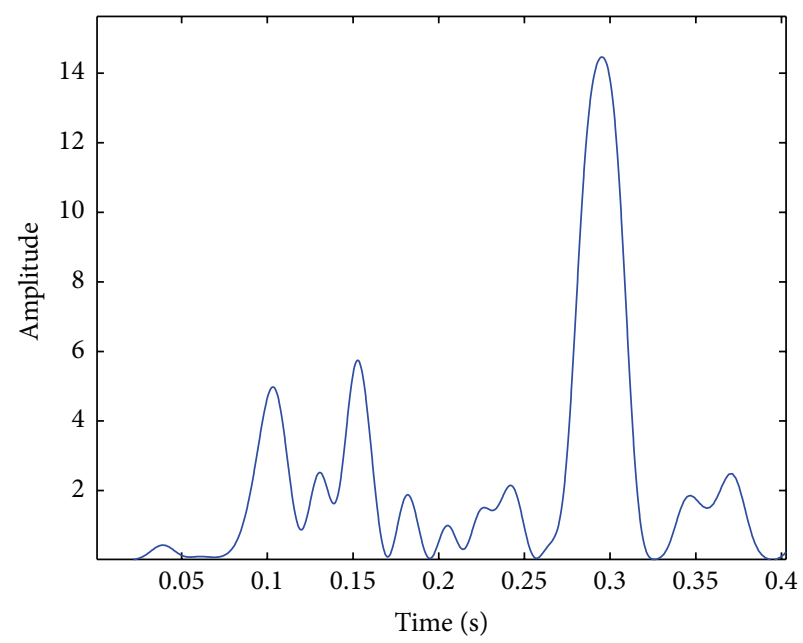

(a) The envelope in time-domain

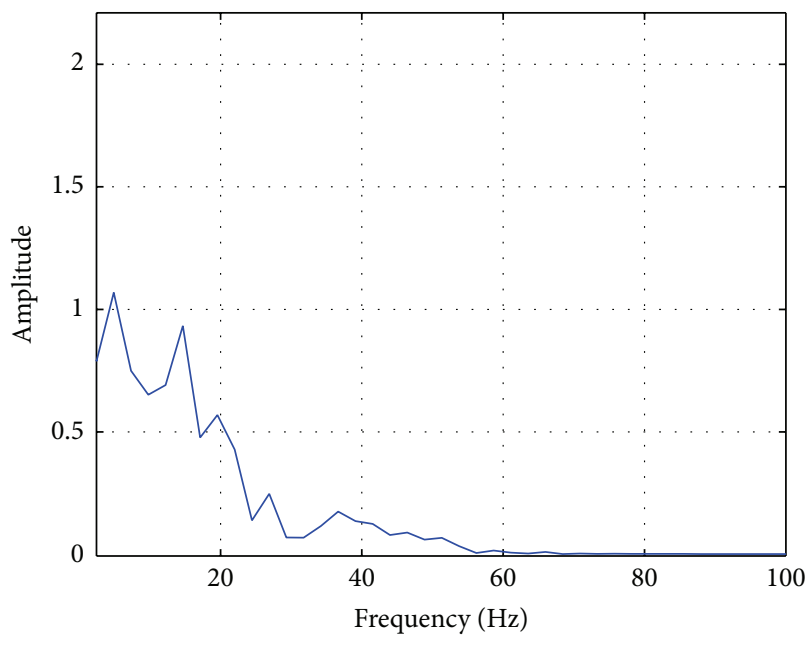

(b) The envelope frequency spectrogram

FIgURE 7: The Hilbert analysis result of contaminated signal with $\mathrm{SNR}=-8 \mathrm{~dB}$.

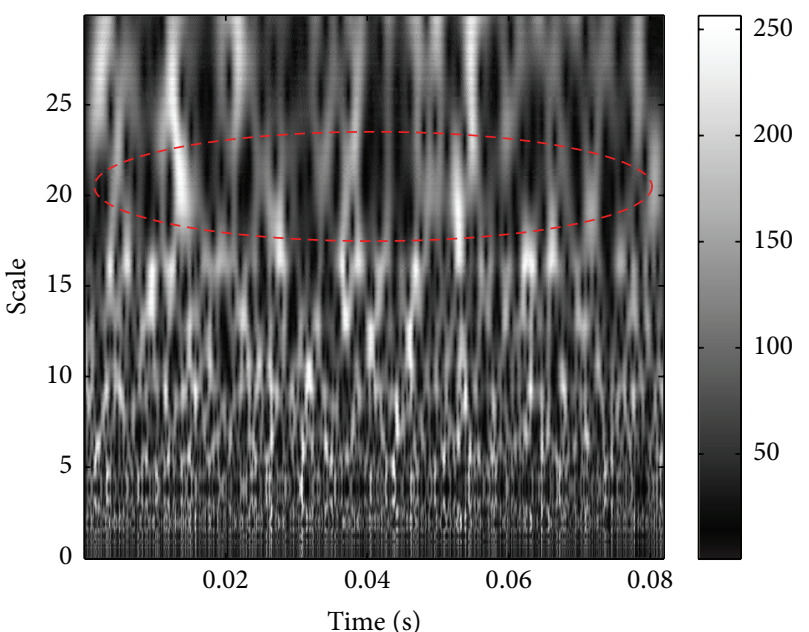

(a) The wavelet envelope on time-scale plane

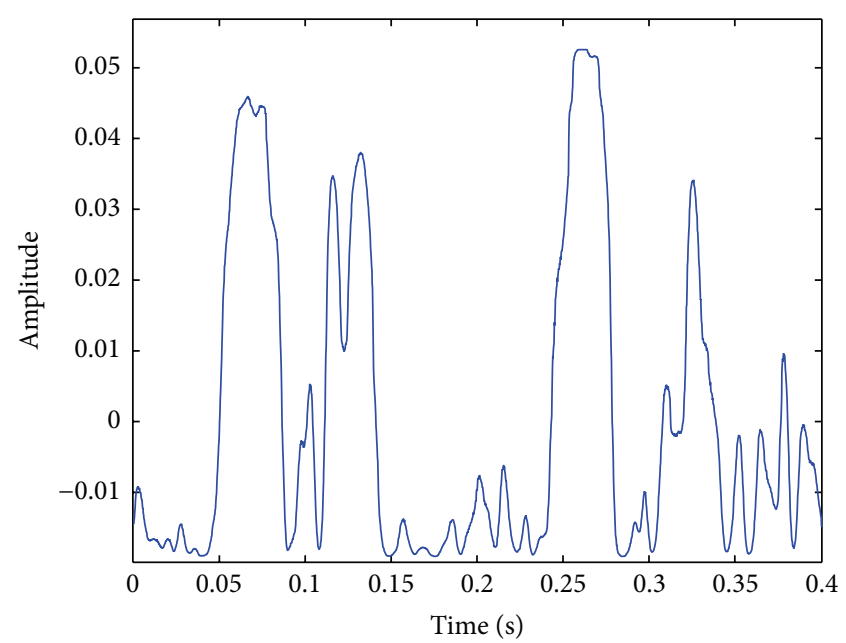

(c) The envelope manifold in time-domain

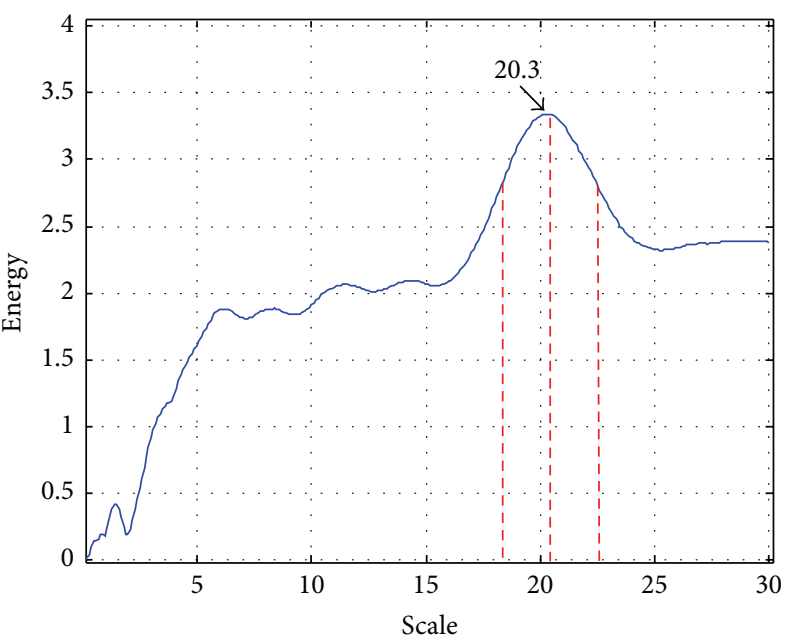

(b) The ME versus scale curve

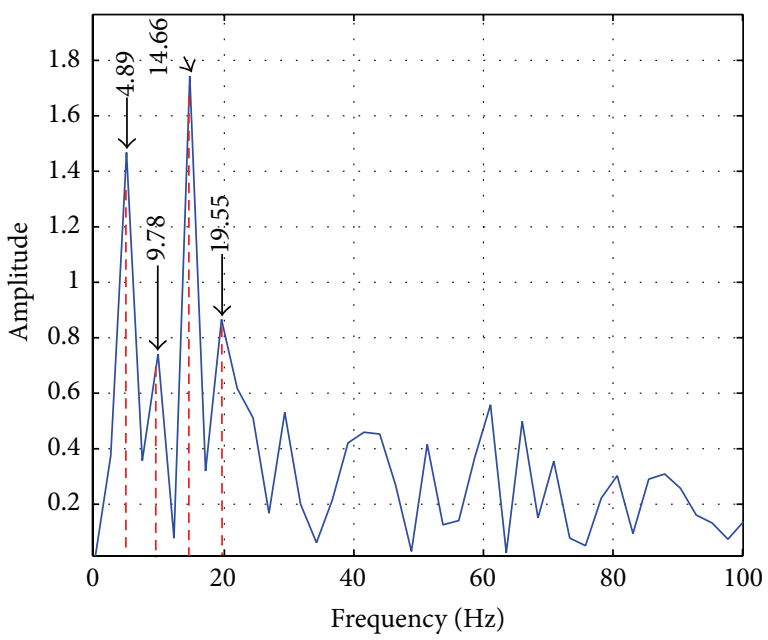

(d) The envelope manifold frequency spectrogram

FIGURE 8: The envelope manifold result of contaminated signal with $\mathrm{SNR}=-10 \mathrm{~dB}$. 


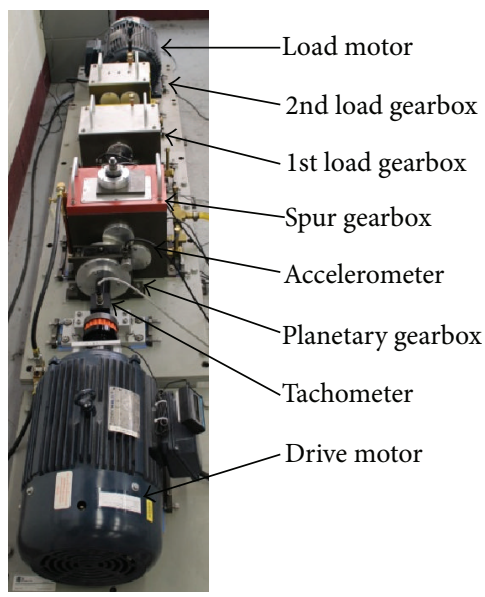

FIgURE 9: The planetary gearbox rig.

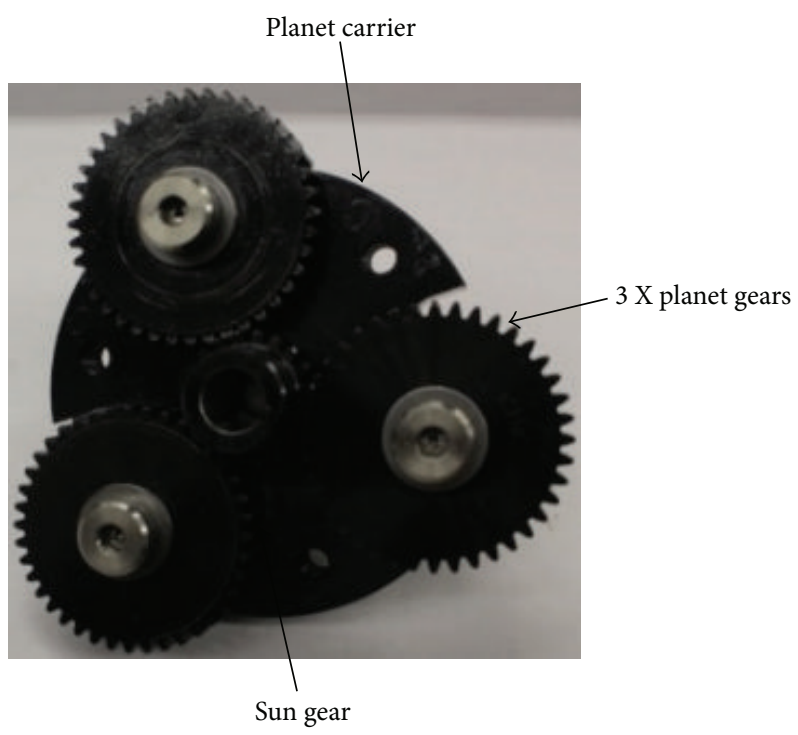

Figure 10: The planetary gear set.

TABLE 3: The working parameters of experiments.

\begin{tabular}{lc}
\hline Parameters & Value $(\mathrm{Hz})$ \\
\hline Sun gear frequency $\left(f_{s}\right)$ & 25 \\
Planet gear meshing frequency $\left(f_{m}\right)$ & 546.88 \\
Planet carrier rotation frequency $\left(f_{\mathrm{ca}}\right)$ & 5.47 \\
Planet gear pass frequency $\left(f_{\mathrm{pp}}\right)$ & 21.88 \\
Planet gear rotation frequency $\left(f_{\mathrm{pr}}\right)$ & 9.72 \\
Faulty planet gear frequency $\left(f_{\mathrm{pf}}\right)$ & 15.19 \\
\hline
\end{tabular}

is the man-made tooth missing planet gear that simulates the local fault, and the third is the teeth surface worn planet gear that simulates the distributed fault.

During experiments, the rotation speed of drive motor is set to $1500 \mathrm{rpm}$. The working parameters of the planetary gearbox in the experiments can be computed which are listed in Table 3. The collected vibration signals in practical experiments are very complicated because of the abundant components in every frequency band. These practical vibration signals are analyzed with envelope manifold demodulation method to extract the fault features properly as follows.

\subsection{Experiment Analysis}

4.2.1. Normal Planet Gear. In order to demonstrate the denoising effect of envelope manifold demodulation at first, the wavelet envelope at the central scale and the envelope manifold are studied for comparison. The central scale is 18.2 according to ME indicator. The corresponding meshing frequency, respectively, is $549.5 \mathrm{~Hz}$. But the central scale, respectively, is 8.0 or 1.8 according to SI or SK. The corresponding frequency, respectively, is $1250 \mathrm{~Hz}$ or $5555.6 \mathrm{~Hz}$. So the ME indicator is most suitable for identifying the planet gear meshing frequency in the practical experiments. 


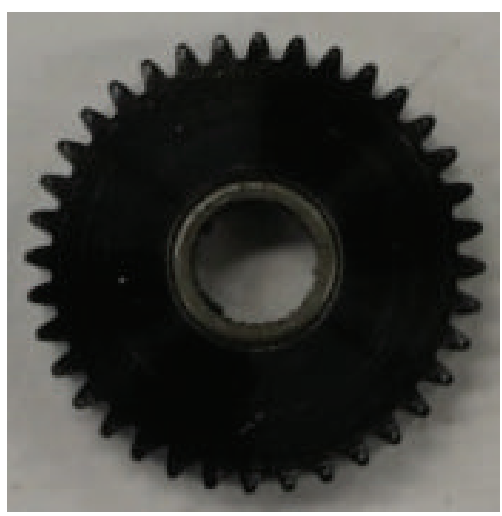

(a)

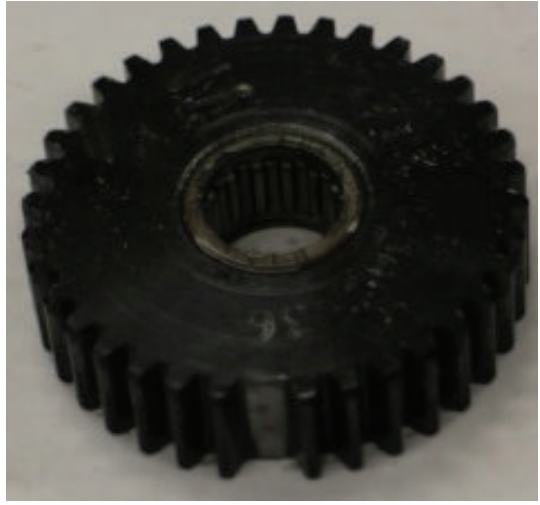

(b)

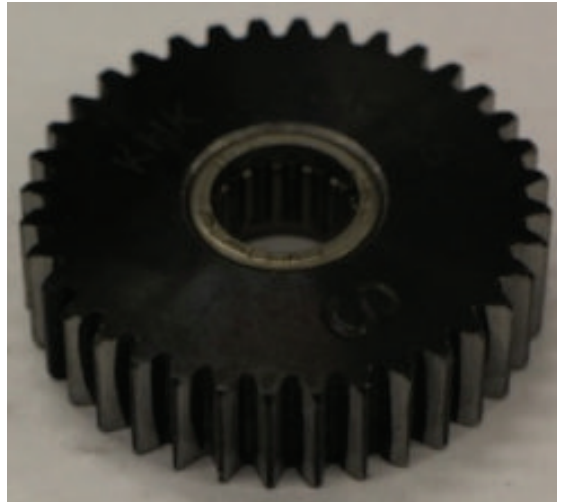

(c)

FIGURE 11: The experimental planet gears.

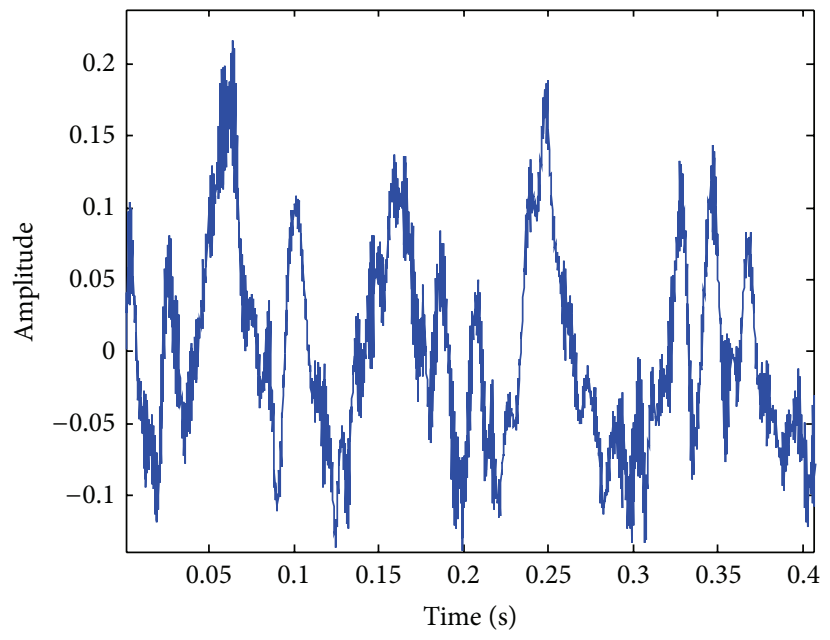

(a) The wavelet envelope in time-domain

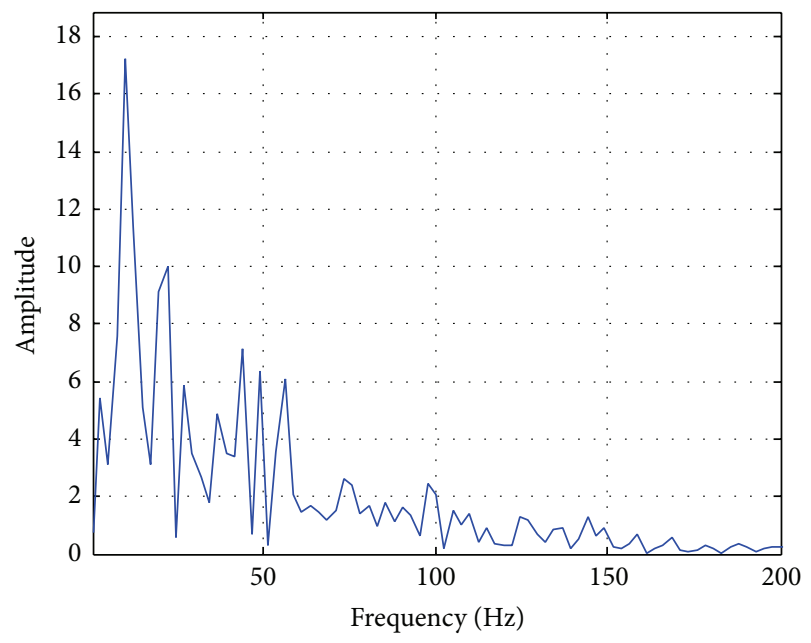

(b) The wavelet envelope frequency spectrogram

Figure 12: The wavelet envelope at the central scale of normal planet gear.

The wavelet envelope at the central scale is shown in Figure 12(a) and the wavelet envelope frequency spectrogram is shown in Figure 12(b). After manifold learning, the envelope manifold and the frequency spectrogram are shown in Figure 13. The in-band noise causes the distortion of wavelet envelope. The characteristic frequency of envelope manifold is clearer than the wavelet envelope by comparing the frequency spectrograms in these figures.

The meshing vibration signal of planetary gearbox is modulated by different meshing phases, varied propagation paths, and even the manufacture error of the gear pairs. So in the envelope manifold spectrogram of the normal planet gear shown in Figure 13(b), the characteristic frequencies of $f_{\mathrm{pp}}, f_{\mathrm{pr}}, f_{\mathrm{pp}}+f_{\mathrm{ca}}, f_{\mathrm{pp}}+2 f_{\mathrm{ca}}, 2 f_{\mathrm{pp}}, 2 f_{\mathrm{pp}}+f_{\mathrm{ca}}, 2 f_{\mathrm{pp}}+2 f_{\mathrm{ca}}, \ldots$ can be recognized. The characteristic frequencies are related to $f_{\mathrm{pp}}, f_{\mathrm{pr}}$, and $f_{\mathrm{ca}}$ but not associated with the faulty planet gear frequency. From spectrogram, we can find the characteristic frequencies caused by the manufacture error, that is, the unbalance of the planet carrier and multiple planet gears.
Their amplitudes are not big. So there is no fault on the planet gears.

4.2.2. Tooth Missing Planet Gear. The envelope manifold of the tooth missing planet gear is shown in Figure 14. The characteristic frequencies of $f_{\mathrm{pf}}, f_{\mathrm{pr}}, f_{\mathrm{pp}}+f_{\mathrm{pf}}, f_{\mathrm{pp}}+f_{\mathrm{ca}}$, $f_{\mathrm{pp}}+2 f_{\mathrm{ca}}, \ldots$ can be seen in the frequency spectrogram. The modulating frequency of faulty planet gear $f_{\mathrm{pf}}$ can be extracted from the demodulated spectrum. $f_{\mathrm{pf}}, f_{\mathrm{pp}}$, and $f_{\mathrm{pr}}$ jointly modulated the meshing frequency of the planet gear. $f_{\text {pf }}$ and $f_{\text {pp }}+f_{\text {pf }}$ become the mainly modulating frequencies besides those characteristic frequencies of normal planet gear. The faulty planet gear frequency and the harmonic frequencies can be distinguished in the frequency spectrogram.

4.2.3. Teeth Surface Worn Planet Gear. The envelope manifold of the teeth surface worn planet gear is shown in Figure 15. The characteristic frequencies of $f_{\mathrm{ca}}, f_{\mathrm{pp}}+f_{\mathrm{pf}}$, 


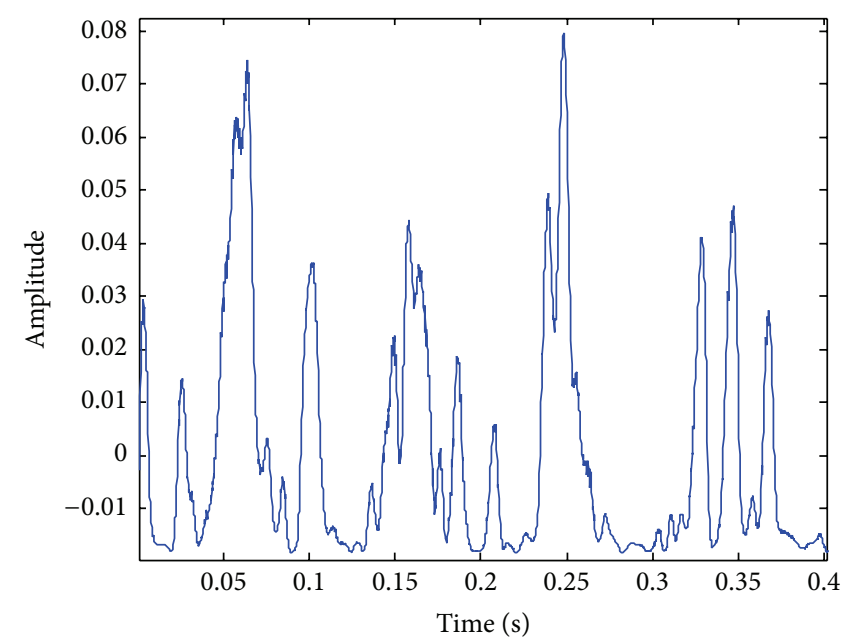

(a) The envelope manifold in time-domain

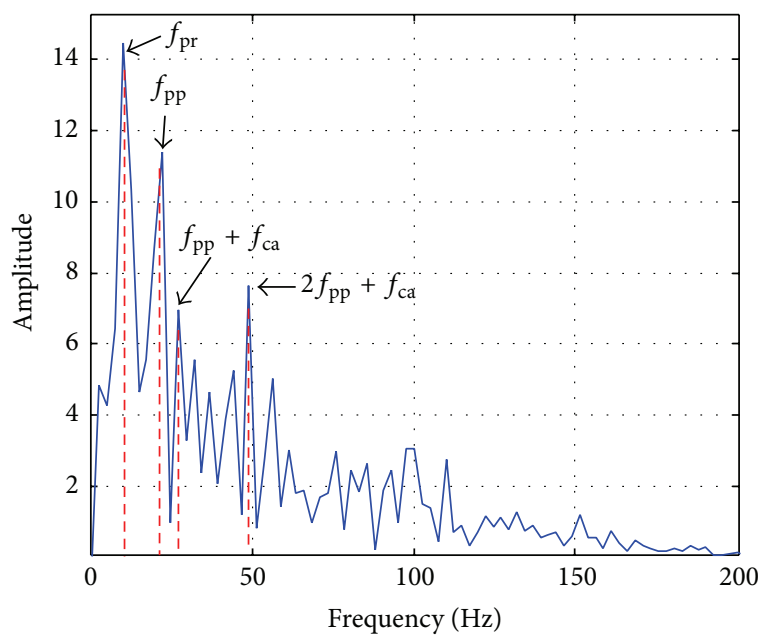

(b) The envelope manifold frequency spectrogram

FIGURE 13: The envelope manifold of normal planet gear.

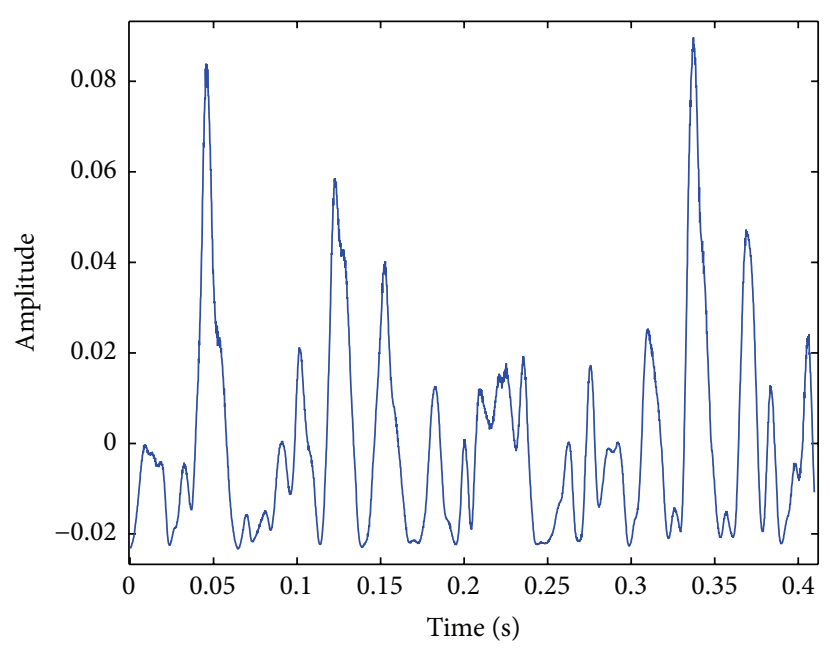

(a) The envelope manifold in time-domain

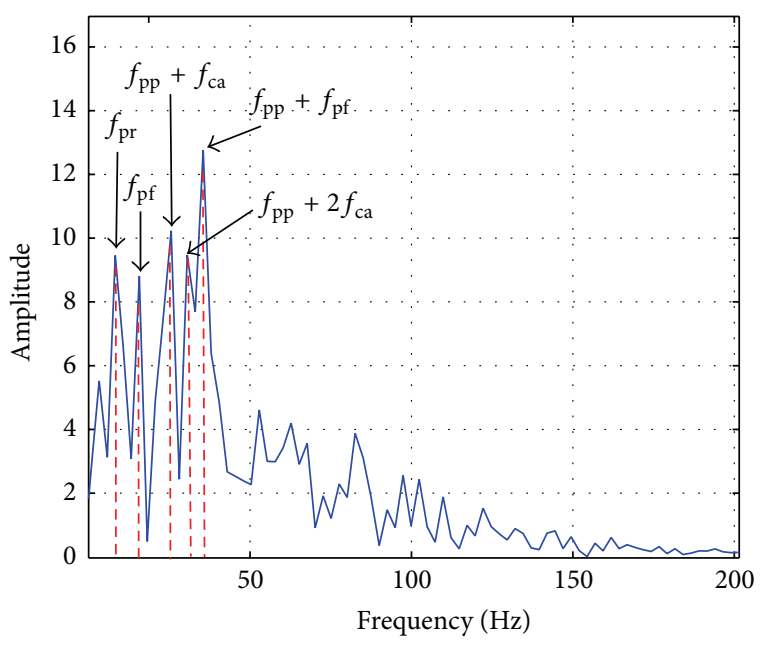

(b) The envelope manifold frequency spectrogram

FIGURE 14: The envelope manifold of tooth missing planet gear.

$f_{\mathrm{pp}}+2 f_{\mathrm{ca}}, f_{\mathrm{pf}}+f_{\mathrm{ca}}, f_{\mathrm{pf}}+f_{\mathrm{pr}}, \ldots$ can be viewed in the frequency spectrogram. Because there is just one teeth surface worn planet gear in the gearbox, the unbalance of the planet carrier increases dramatically. The effect of planet carrier characteristic frequency is enhanced. When the teeth surface worn planet gear meshes with mating gears, it can cause the faulty planet gear characteristic frequency. And so the frequencies of $f_{\mathrm{pf}}+f_{\mathrm{pr}}$ and $f_{\mathrm{pf}}+f_{\mathrm{ca}}$ appear in the power spectrogram. The teeth surface worn planet gear can be identified by this way.

The planetary gearbox rig sets and experiment conditions are different from each other. All the experiment results are based on the special rig set and experiment condition, but the experiments validate the effectiveness of the envelope manifold demodulation methodology.

\section{Conclusions}

In this paper, the envelope manifold demodulation methodology was introduced to implement the fault diagnosis of planetary gearbox. The method consisted of the complex wavelet transform, manifold learning, and frequency spectrogram analysis method. The continuous wavelet transform with complex Morlet wavelet was utilized to extract the envelope for demodulation of the planetary gears meshing vibration signal at first. The scale band selection was actualized through mean envelope energy (ME) indicator to locate the meshing frequency and remove the noisy signal not within the meshing frequency band. Then, the manifold learning method is applied to extract the intrinsic manifold of the envelope and reduce the effect of in-band noise. At last, 


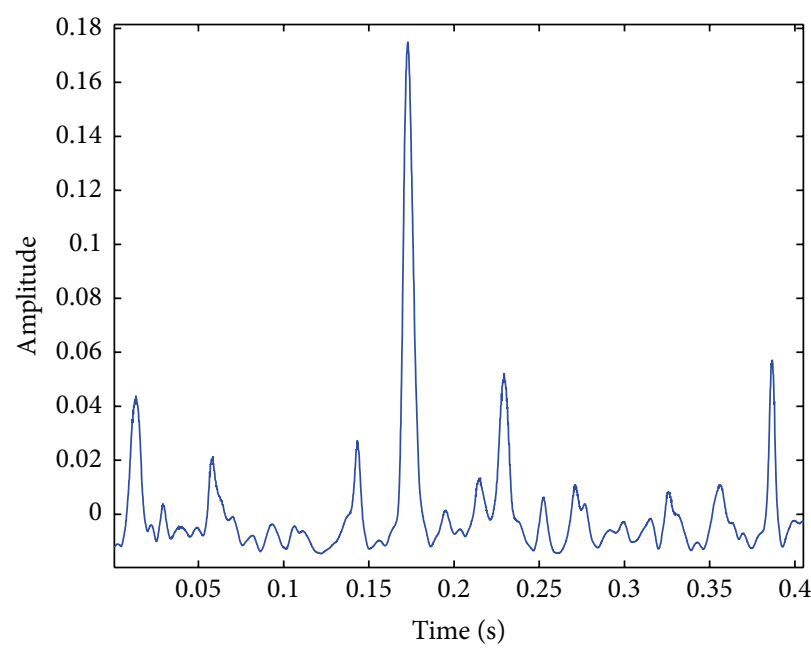

(a) The envelope manifold in time-domain

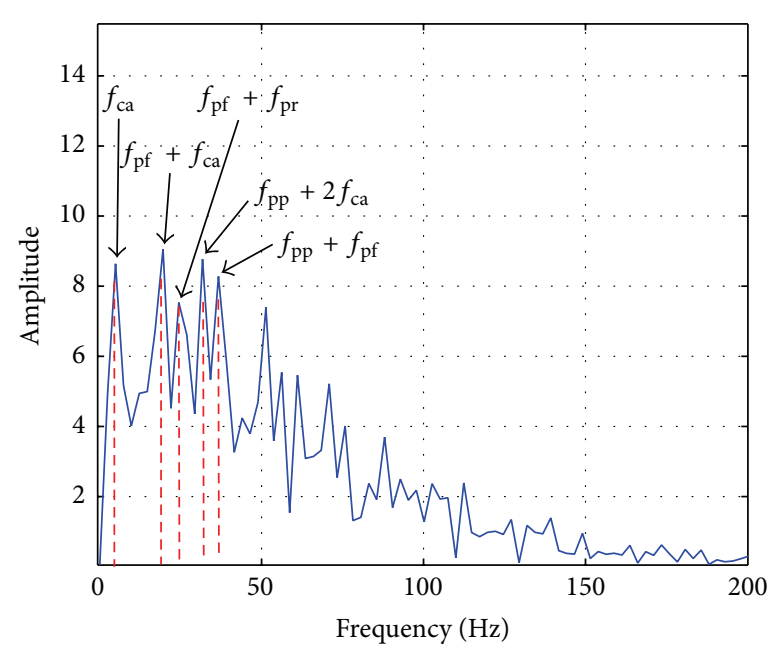

(b) The envelope manifold frequency spectrogram

FIGURE 15: The envelope manifold of teeth surface worn planet gear.

the frequency spectrogram is used to show the characteristic frequency of the faulty planetary gears meshing envelope. By this method, the effect of noise among the vibration signals was immensely weakened, the envelope manifold structure was clearly revealed, and the fault characteristics of planetary gearbox were conveniently extracted. The result of simulated signal and experimental dataset all verified the effectiveness of the method. This method revealed the intrinsic characteristics of the planetary gearbox vibration signal. It was observed that this method outperformed other approaches in various conditions. The further work of us will be research on the exact mechanism of the planetary gearbox vibrations based on the results of this paper.

\section{Conflict of Interests}

The authors declare that there is no conflict of interests regarding the publication of this paper.

\section{Acknowledgments}

This project is supported by the National Natural Science Foundation of China (51275030) and the Fundamental Research Funds for the Central Universities (2011JBM093).

\section{References}

[1] J. McNames, "Fourier series analysis of epicyclic gearbox vibration," Journal of Vibration and Acoustics, vol. 124, no. 1, pp. 150152, 2002.

[2] J. L. Chen, Y. Y. Zi, Z. J. He, and X. D. Wang, "Construction of adaptive redundant multiwavelet packet and its application to compound faults detection of rotating machinery," Science China Technological Sciences, vol. 55, no. 8, pp. 2083-2090, 2012.

[3] H. L. Sun, Y. Y. Zi, Z. J. He, J. Yuan, X. Wang, and L. Chen, "Customized multiwavelets for planetary gearbox fault detection based on vibration sensor signals," Sensors, vol. 13, no. 1, pp. 1183-1209, 2013.
[4] Y. G. Lei, D. Han, J. Lin, and Z. He, "Planetary gearbox fault diagnosis using an adaptive stochastic resonance method," Mechanical Systems and Signal Processing, vol. 38, no. 1, pp. 113124, 2013.

[5] Y. G. Lei, J. Lin, M. J. Zuo, and Z. J. He, “Condition monitoring and fault diagnosis of planetary gearboxes: a review," Measurement, vol. 48, no. 2, pp. 292-305, 2014.

[6] F. Chaari, T. Fakhfakh, and M. Haddar, "Analytical investigation on the effect of gear teeth faults on the dynamic response of a planetary gear set," Noise \& Vibration Worldwide, vol. 37, no. 8, pp. 9-15, 2006.

[7] Z. Cheng and N. Q. Hu, "Quantitative damage detection for planetary gear sets based on physical models," Chinese Journal of Mechanical Engineering, vol. 25, no. 1, pp. 190-196, 2012.

[8] Z. Feng and M. J. Zuo, "Vibration signal models for fault diagnosis of planetary gearboxes," Journal of Sound and Vibration, vol. 331, no. 22, pp. 4919-4939, 2012.

[9] P. D. McFadden, "A technique for calculating the time domain averages of the vibration of the individual planet gears and the sun gear in an epicyclic gearbox," Journal of Sound and Vibration, vol. 144, no. 1, pp. 163-172, 1991.

[10] Y. G. Lei, D. T. Kong, J. Lin, and M. J. Zuo, "Fault detection of planetary gearboxes using new diagnostic parameters," Measurement Science and Technology, vol. 23, no. 5, Article ID 055605, 2012.

[11] W. D. Mark, "Stationary transducer response to planetarygear vibration excitation II: effects of torque modulations," Mechanical Systems and Signal Processing, vol. 23, no. 7, pp. 2253-2259, 2009.

[12] F. Chaari, T. Fakhfakh, and M. Haddar, "Dynamic analysis of a planetary gear failure caused by tooth pitting and cracking," Journal of Failure Analysis and Prevention, vol. 6, no. 2, pp. 7378, 2006.

[13] Y. Jiang, B. Tang, Y. Qin, and W. Liu, "Feature extraction method of wind turbine based on adaptive Morlet wavelet and SVD," Renewable Energy, vol. 36, no. 8, pp. 2146-2153, 2011.

[14] T. Barszcz and R. B. Randall, "Application of spectral kurtosis for detection of a tooth crack in the planetary gear of a wind 
turbine," Mechanical Systems and Signal Processing, vol. 23, no. 4, pp. 1352-1365, 2009.

[15] R. Zimroz and W. Bartelmus, "Gearbox condition estimation using cyclostationary properties of vibration signal," Key Engineering Materials, vol. 413-414, no. 1, pp. 471-478, 2009.

[16] J. Zheng, J. Cheng, and Y. Yang, "Multi-scale permutation entropy based rolling bearing fault diagnosis," Shock and Vibration, vol. 2014, Article ID 154291, 8 pages, 2014.

[17] W. Caesarendra, B. Kosasih, A. K. Tieu, and C. A. S. Moodie, "Application of the largest Lyapunov exponent algorithm for feature extraction in low speed slew bearing condition monitoring," Mechanical Systems and Signal Processing, vol. 50-51, no. 1, pp. 116-138, 2015.

[18] R. Q. Yan and R. X. Gao, "Approximate entropy as a diagnostic tool for machine health monitoring," Mechanical Systems and Signal Processing, vol. 21, no. 2, pp. 824-839, 2007.

[19] W. J. Wang, Z. T. Wu, and J. Chen, "Fault identification in rotating machinery using the correlation dimension and bispectra," Nonlinear Dynamics, vol. 25, no. 4, pp. 383-393, 2001.

[20] J. Wang, Q. B. He, and F. R. Kong, "Multiscale envelope manifold for enhanced fault diagnosis of rotating machines," Mechanical Systems and Signal Processing, vol. 52-53, no. 1, pp. 376-392, 2015.

[21] Q. He, Y. Liu, Q. Long, and J. Wang, “Time-frequency manifold as a signature for machine health diagnosis," IEEE Transactions on Instrumentation and Measurement, vol. 61, no. 5, pp. 12181230, 2012.

[22] J. Wang, Q. B. He, and F. R. Kong, "Automatic fault diagnosis of rotating machines by time-scale manifold ridge analysis," Mechanical Systems and Signal Processing, vol. 40, no. 1, pp. 237256, 2013

[23] J. Wang and Q. B. He, "Exchanged ridge demodulation of time-scale manifold for enhanced fault diagnosis of rotating machinery," Journal of Sound and Vibration, vol. 333, no. 11, pp. 2450-2464, 2014.

[24] D. M. Blunt and J. A. Keller, "Detection of a fatigue crack in a UH-60A planet gear carrier using vibration analysis," Mechanical Systems and Signal Processing, vol. 20, no. 8, pp. 2095-2111, 2006.

[25] R. Q. Yan and R. X. Gao, "Multi-scale enveloping spectrogram for vibration analysis in bearing defect diagnosis," Tribology International, vol. 42, no. 2, pp. 293-302, 2009.

[26] J. Antoni, "The spectral kurtosis: a useful tool for characterising non-stationary signals," Mechanical Systems and Signal Processing, vol. 20, no. 2, pp. 282-307, 2006.

[27] X. H. Zhang, J. S. Kang, L. Xiao, J. Zhao, and H. Teng, "A new improved kurtogram and its application to bearing fault diagnosis," Shock and Vibration, vol. 2015, Article ID 385412, 22 pages, 2015.

[28] Y. X. Wang and M. Liang, "Identification of multiple transient faults based on the adaptive spectral kurtosis method," Journal of Sound and Vibration, vol. 331, no. 2, pp. 470-486, 2012.

[29] P. W. Tse and D. Wang, "The design of a new sparsogram for fast bearing fault diagnosis: part 1 of the two related manuscripts that have a joint title as 'two automatic vibration-based fault diagnostic methods using the novel sparsity measurementparts 1 and 2"' Mechanical Systems and Signal Processing, vol. 40, no. 2, pp. 499-519, 2013.

[30] I. S. Bozchalooi and M. Liang, "A joint resonance frequency estimation and in-band noise reduction method for enhancing the detectability of bearing fault signals," Mechanical Systems and Signal Processing, vol. 22, no. 4, pp. 915-933, 2008.
[31] S. T. Roweis and L. K. Saul, "Nonlinear dimensionality reduction by locally linear embedding," Science, vol. 290, no. 5500, pp. 2323-2326, 2000.

[32] J. B. Tenenbaum, V. De Silva, and J. C. Langford, "A global geometric framework for nonlinear dimensionality reduction," Science, vol. 290, no. 5500, pp. 2319-2323, 2000.

[33] A. J. Izenman, "Introduction to manifold learning," Wiley Interdisciplinary Reviews: Computational Statistics, vol. 4, no. 5, pp. 439-446, 2012.

[34] M. Belkin and P. Niyogi, "Laplacian eigenmaps and spectral techniques for embedding and clustering," in Advances in Neural Information Processing Systems, vol. 14, pp. 585-591, MIT Press, 2002.

[35] S. Natarajan, "Gear box fault diagnosis using hilbert transform and study on classification of features by support vector machine," International Journal of Hybrid Information Technology, vol. 7, no. 4, pp. 69-82, 2014.

[36] P. Flandrin, G. Rilling, and P. Gonçalvés, "Empirical mode decomposition as a filter bank," IEEE Signal Processing Letters, vol. 11, no. 2, pp. 112-114, 2004. 


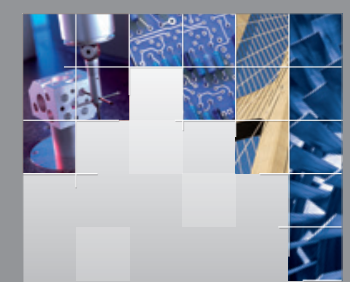

\section{Enfincering}
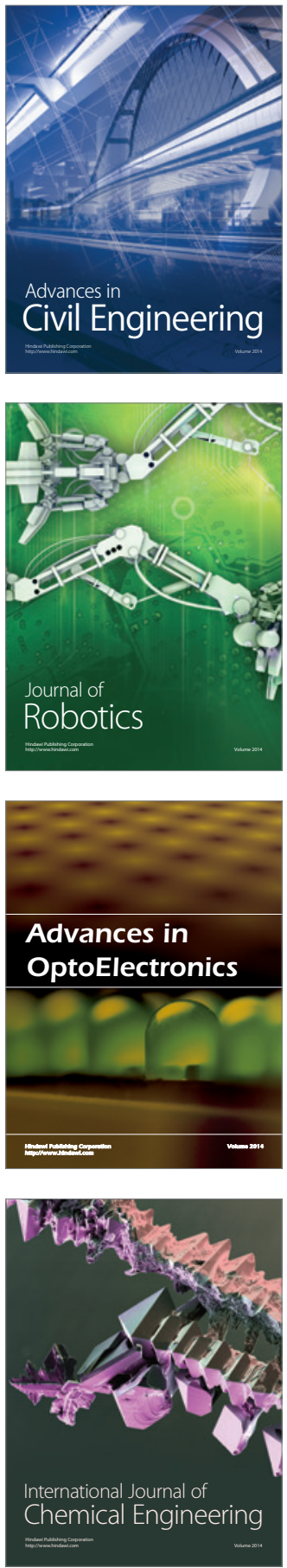

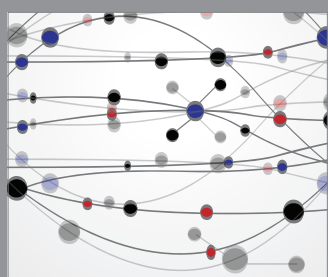

The Scientific World Journal

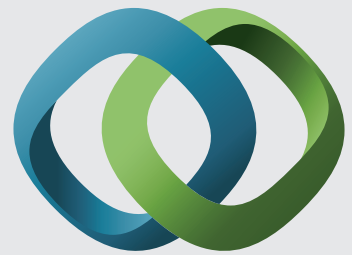

\section{Hindawi}

Submit your manuscripts at

http://www.hindawi.com
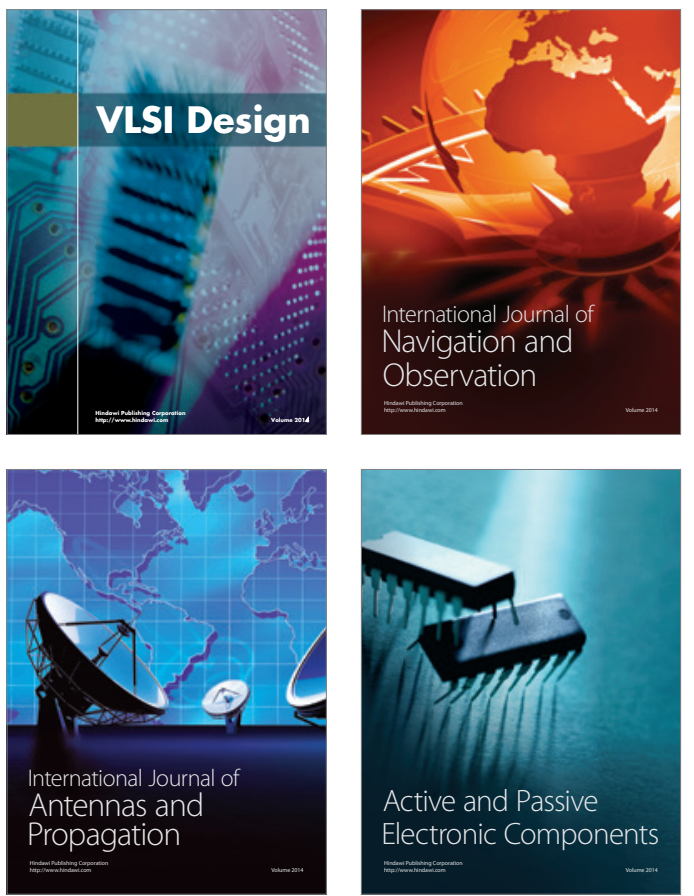
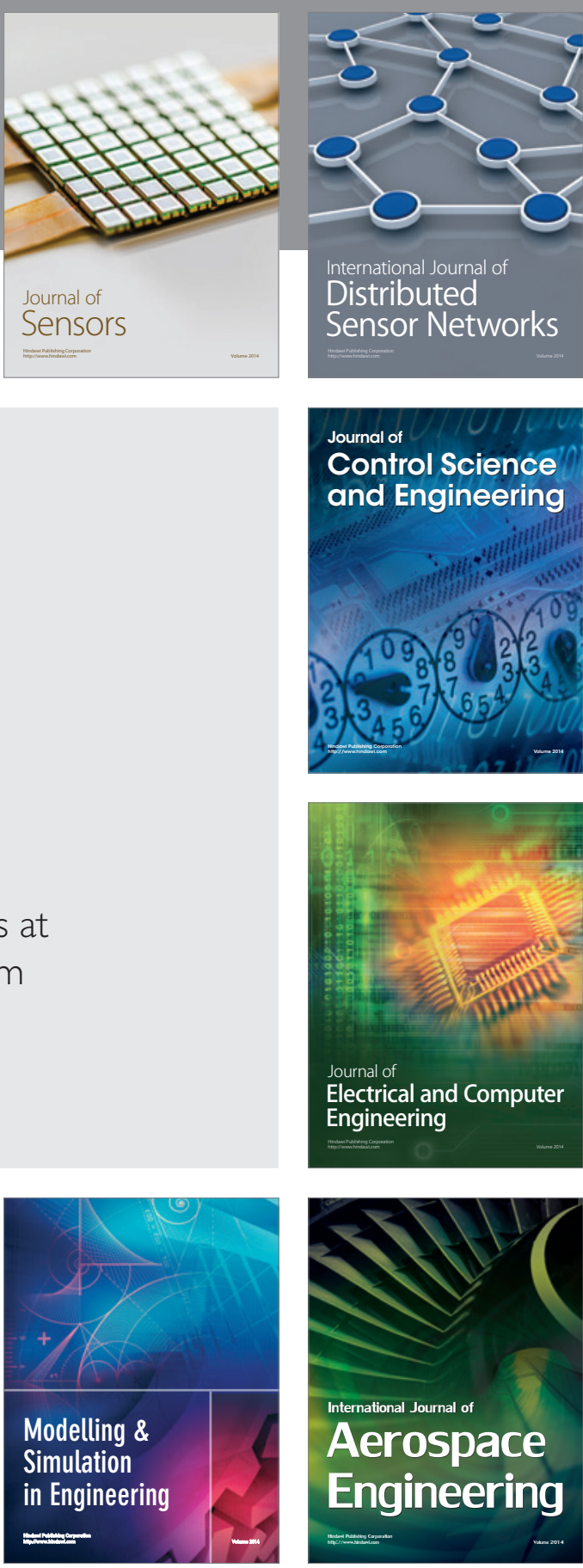

International Journal of

Distributed

Sensor Networks

Journal of

Control Science

and Engineering
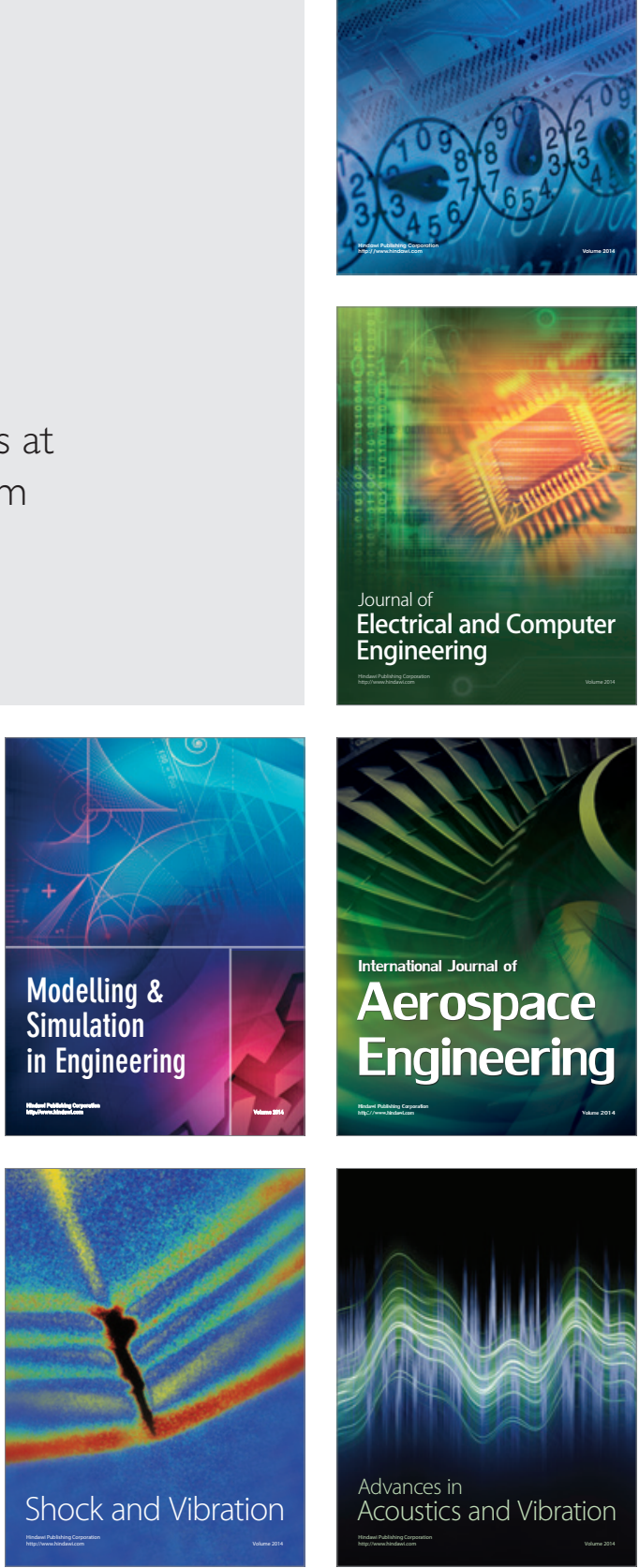